\title{
A CALORIMETRIC INVESTIGATION OF MARTENSITIC TRANSFORMATION UNDIER APPLIED STRESS IN SINGLE-CRYSTAL Cu-AL-NI ALLOYS
}

\author{
C.M. FRIEND, Ll. MAŇOSA*, J. ORTÍN* and A. PLANES* \\ Materials Technology Group, Cranfield Institute of Technology, Shrivenham Campus, Swindon, \\ Great-Britain \\ Departament d'Estructura $i$ Constituent de la Matèria, Faculta de Fisica, Universitat de Barcelona, \\ Catalonia, Spain
}

\begin{abstract}
A high sensitivity microcalorimeter is described which is capable of carrying out measurements on samples subjected to uniaxial tensile stress. This novel calorimeter was used, together with stress-free calorimetry, to map the composition dependence of the martensite phase-stabilities in four $\mathrm{Cu}-\mathrm{Al}-\mathrm{Ni}$ alloys. It is shown that stress-temperature phase diagrams produced using this approach are extremely interesting since they contain phase boundaries which were previously inaccessible during stress-induced transformation experiments. Direct calorimetric determinations of the enthalpy and entropy changes during $B \rightarrow \gamma^{\prime}$ transformation are also presented for the four alloys, and the effect of applied stress on the enthalpy and entropy changes during both $\beta \rightarrow \gamma^{\prime}$, and $\beta \rightarrow \beta^{\prime}$ transformation are analysed for one of the alloys.
\end{abstract}

\section{INTRODUCTION}

Noble metal-based alloys with bcc structures ( $\beta$-phases) undergo a number of different thermoelastic martensitic transformations. The relative stabilities of these different martensitic phases depend not only on temperature and alloy composition, but also on the level of externally applied stress /1/. This leads to relatively complex stress/temperature equilibria. The objective of much research on these alloys has been the determination of the enthalpy and entropy changes associated with these transformations. The usual procedure for such investigations is to experimentally determine the stress/temperature phase diagram for a given alloy by isothermal tensile testing. The entropies of transformation can then be determined from the temperature dependencies of the transformational stress-thresholds using the clausius-clapeyron equation, and the enthalpies of transformation (at the equilibrium temperatures between the phases, $T_{0}$ ) calculated using this entropy change and an estimated value for $T_{0}$. However, an alternative approach to the evaluation of this data is available. This is to perform direct calorimetric measurements on thermally-induced transformations in samples subjected to applied stress.

The aim of this paper is (i) to describe an experimental apparatus which can carry out such direct calorimetric measurements, (ii) to present examples of stress/temperature phase diagrams determined using this approach, and (iii) to report direct calorimetric determinations of the enthalpy and entropy changes during $B \rightarrow \gamma^{\prime}$ transformation in four $\mathrm{Cu}-\mathrm{Al}-\mathrm{N} i$ 
alloys, and for one of these alloys an evaluation of the effect of applied stress on the enthalpy and entropy changes during both the $B \rightarrow \gamma^{\prime}$ and $B$ $\rightarrow B^{\prime}$ transformations.

\section{EXPERIMENTAL APPARATUS.}

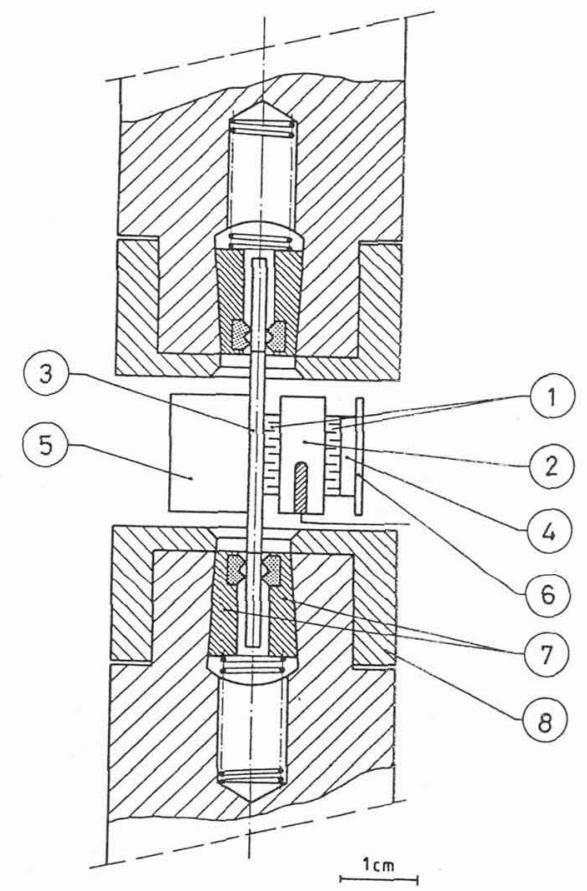

Fig 1-Microcalorimeter
The central part of the experimental apparatus was the scanning microcalorimeter shown in fig 1 . This consisted of two semi-conducting bismuth telluride thermobatteries (connected in differential) (1) separated by a copper calorimetric block (2). One thermobattery was in contact with the flat tensile specimens (3), and the temperature of the calorimeter was measured by a four wire Pt-100 resistance embedded in the copper block. This microcalorimeter was located inside a cylindrical heating and cooling chamber and the heads of the tensile specimens were held by two grips which ran axially through this chamber. These grips could either be mounted in a conventional tensile test machine, or alternatively connected to a dead-weight loading mechanism. The calorimetric signal and that from the thermometer were sequentially sampled by a microcomputer which controlled both the operation of the apparatus and data acquisition. The calorimetric sensitivity was about 170 $\mathrm{mVW}^{-1}$ at room temperature $/ 2 /$. Such a sensitivity enabled calorimetric measurements during both isothérmal stress-induced transformations, and thermally-induced transformations at constant stress. The data treatment required for both types of measurement has been described previously $/ 2,3 /$.

\section{EXPERIMENTAL TECHNIQUES.}

Calorimetric measurements were carried out on four single-crystal Cu-Al-Ni alloys. The compositions of these alloys are shown in Table 1.

TABLE 1 - Alloy Compositions (at\%)

\begin{tabular}{||c|c|c|c|c|}
\hline & A & B & C & D \\
\hline Cu & 68.9 & 68.4 & 67.9 & 67.4 \\
$\mathrm{Al}$ & 27.3 & 27.8 & 28.3 & 28.8 \\
$\mathrm{Ni}$ & 3.8 & 3.8 & 3.8 & 3.8 \\
\hline
\end{tabular}

The preparation and characterisation of the specimens have been described in two previous papers /2,4/. Calorimetric measurements were carried out in the apparatus described above using two distinct transformation routes. These were (i) isothermal stress-induced transformation, and (ii) thermally-induced transformation under constant stress. Stress-free calorimetric measurements were also carried out using a second heatconduction scanning microcalorimeter. The details of this apparatus have also been described elsewhere /5/. 


\section{RESULTS AND DISCUSSION.}

Preliminary stress-temperature phase diagrams for the four alloys are shown in figs $2,3,4$, and 5 .

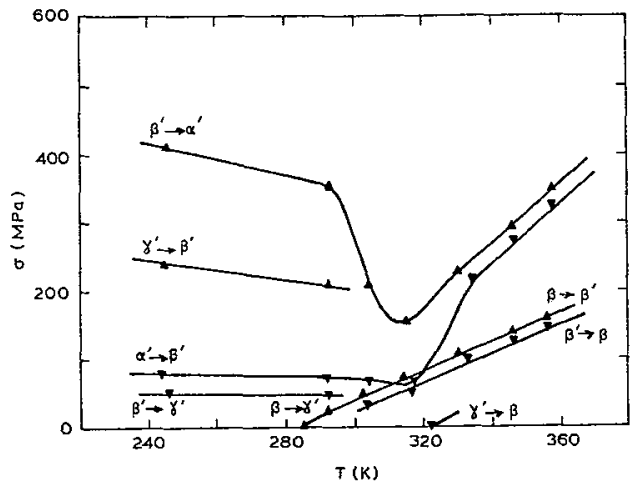

Fig $2-\sigma / \mathrm{T}$ diagram for alloy $\mathrm{A}$

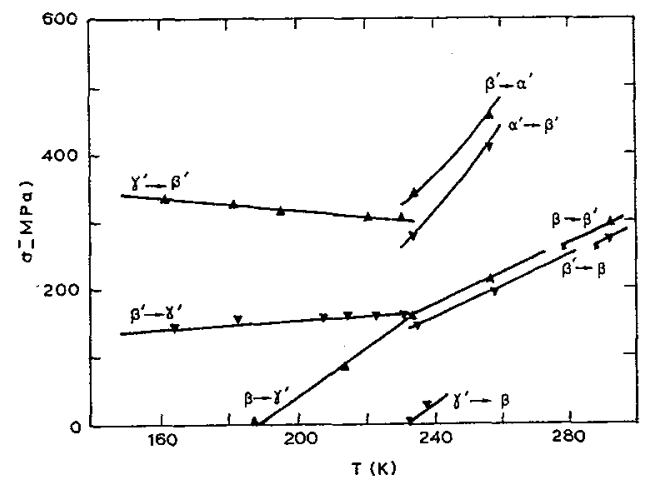

Fig $4-\sigma / T$ diagram for alloy $C$

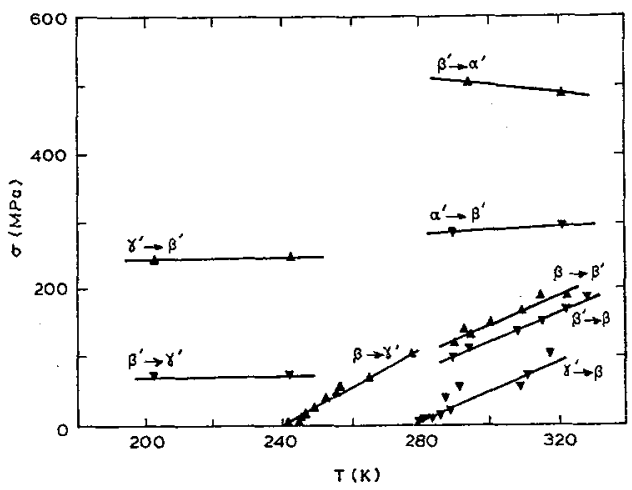

Fig 3- $\sigma / \mathrm{T}$ diagram for alloy $\mathrm{B}$

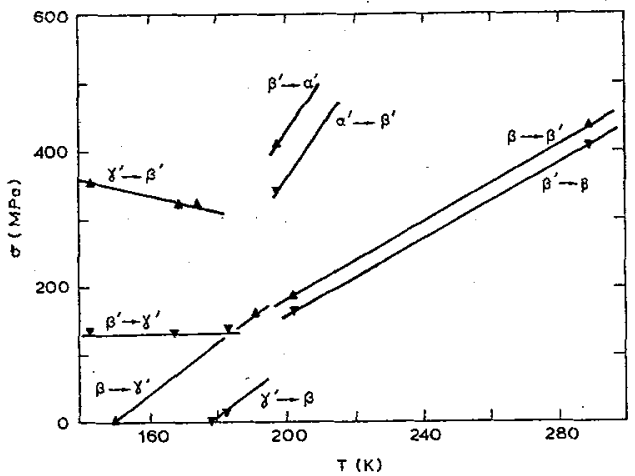

Fig $5-\sigma / T$ diagram for alloy $\mathrm{C}$

The general forms of these diagrams are consistent with established results /6,7/ for $\mathrm{Cu}-\mathrm{Al}-\mathrm{Ni}$ alloys with similar compositions, however, they also illustrate a number of new features:

(i) In addition to the traditional stress-induced data, these diagrams also contain data from thermally-induced transformations at constant stress. Data derived from both transformation routes fall on a single phase boundary which confirms the behaviour expected of a first-order transition.

(ii) The ability to thermally induce transformation at constant stress enabled the determination of the $\gamma^{\prime} \rightarrow B$ phase boundaries. This is extremely important since this reverse transformation is usually 
inaccessible during isothermal stress-induced measurements due to the large hysteresis exhibited by the transformation.

(iii) In two of the four alloys there were anomalous inflections in the $B^{\prime} \rightarrow \alpha^{\prime}$ martensite to martensite transformation thresholds, indicating the possibility of reentrant phases.

The data acquired during these measurements enabled the determination of the entropies and enthalpies of transformation. Stress-free transformation in these four alloys resulted in the formation of $\gamma^{\prime}$ martensite, and thermograms from such transformations have been presented in a previous paper $/ 4 /$. Analysis of these measurements produced the thermodynamic quantities summarised in table 2 .

\section{TABLE 2 - Thermodynamic Data from} Stress-Free Calorimetry

\begin{tabular}{||l|r|r|r|r|r||}
\hline \hline$A L L O Y$ & \multicolumn{1}{|c|}{$\mathrm{A}$} & $\mathrm{B}$ & \multicolumn{1}{c|}{$\mathrm{C}$} & \multicolumn{1}{c|}{$\mathrm{D}$} \\
\hline $\mathrm{M}_{\mathrm{s}}(\mathrm{K})$ & $284 \pm 2$ & $242 \pm 2$ & $188 \pm 1$ & $151 \pm 1$ \\
$\mathrm{~A}_{\mathrm{f}}(\mathrm{K})$ & $322 \pm 2$ & $281 \pm 1$ & $224 \pm 1$ & $177 \pm 2$ \\
$\Delta \mathrm{H}^{\beta+\gamma^{\prime}}\left(\mathrm{Jmol}^{-1}\right)$ & $-407 \pm 14$ & $-371 \pm 13$ & $-303 \pm 1$ & $-227 \pm 1$ \\
$\Delta \mathrm{H}^{\gamma^{\prime \rightarrow \beta}}\left(\mathrm{Jmol}^{-1}\right)$ & $395 \pm 10$ & $326 \pm 20$ & $299 \pm 1$ & $223 \pm 1$ \\
$\Delta \mathrm{S}^{\beta+\gamma^{\prime}}\left(\mathrm{Jmol}^{-1} \mathrm{~K}^{-1}\right)$ & $-1.46 \pm 0.06$ & $-1.54 \pm 0.07$ & $-1.61 \pm 0.01$ & $-1.50 \pm 0.01$ \\
$\Delta \mathrm{S}^{\gamma^{\prime \rightarrow \beta}}\left(\mathrm{Jmol}^{-1} \mathrm{~K}^{-1}\right)$ & $1.31 \pm 0.04$ & $1.18 \pm 0.07$ & $1.35 \pm 0.01$ & $1.27 \pm 0.01$ \\
\hline
\end{tabular}

These enthalpies are consistent with those obtained previously in this family of alloys by both DSC measurements $/ 8 /$ and calculation $/ 9 /$, and their composition dependence is also consistent with previously calculated results $/ 9 /$. The results in table 2 indicate that within experimental errors the entropy changes during transformation were independent of composition, and that there were systematic differences between the absolute values of the entropies for forward $(p \rightarrow m)$ and reverse $(m \rightarrow p)$ transformation. The enthalpy changes exhibited a linear dependence on composition (which is in agreement with previous data /1/) except for the data from alloy $A$. This anomaly has been analysed in a previous paper /4/ where it was shown to be due to the alloy transforming to a mixture of $\gamma^{\prime}$ and $B^{\prime}$ martensites.

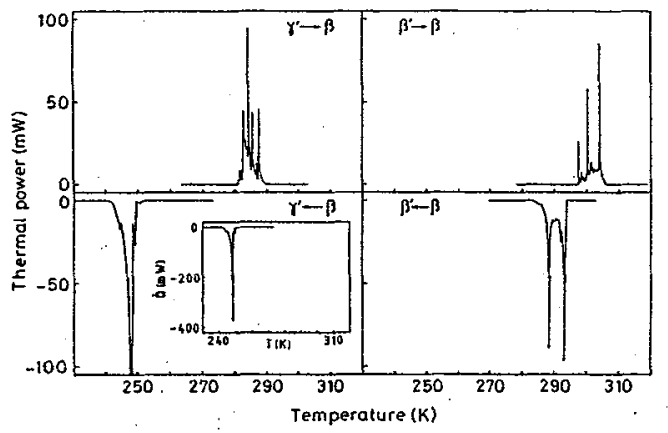

Fig 6-Thermograms for alloy B (a) $22 \mathrm{MPa}$ (b) $142 \mathrm{MPa}$

Figure 6 shows typical thermograms generated from a specimen subjected to applied stress. At low stresses (fig 6a) transformation was to $\gamma^{\prime}$ and produced thermograms similar to those obtained during stress-free measurements. However, at higher stresses the thermally-induced martensite changed to $B^{\prime}$ (as can be seen from the narrower transformation hysteresis). Using the data analysis described in a previous paper /3/ it was therefore possible to calculate direct values for the entropies and enthalpies for both $B \rightarrow \gamma^{\prime}$ and $B \rightarrow B^{\prime}$ transformation under different loads. This was investigated for alloy $B$. Within the experimental accuracy the entropy changes for both transformations were independent of applied stress. However, figure 7 shows that the exchanged heats (Q) increased with applied stress. This is in good agreement with theoretical predictions /3,10/. Extrapolating these 


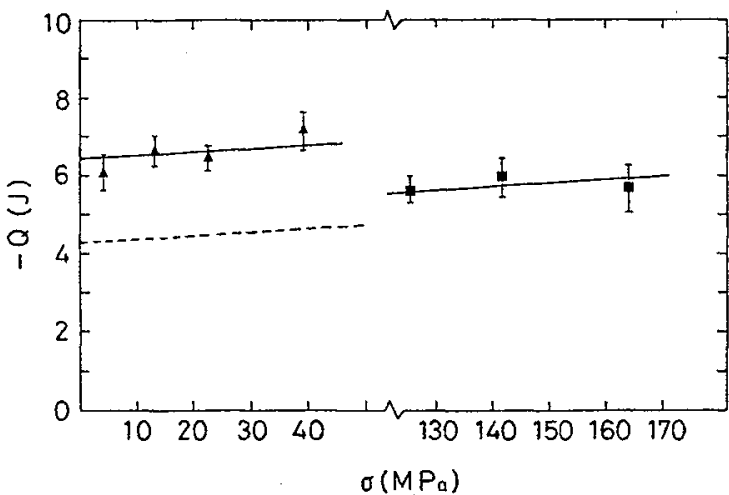

Fig 7-Effect of applied stress on the exchanged heats curves to zero stress allows the enthalpy changes $(Q$ at $\sigma=0)$ of both transformations to be determined. Such a procedure gives values of $-6.5 \pm 0.2$ and $-4.3 \pm$ $0.3 \mathrm{~J}$ for the total magnitudes of $\Delta \mathrm{H}^{\mathrm{B}} \rightarrow \gamma^{\prime}$ and $\Delta \mathrm{H}^{\mathrm{B}} \rightarrow \mathrm{B}^{\prime}$. It is more difficult to determine the molar magnitudes of these quantities since the amount of transformed material within a tensile specimen is unknown. This problem was addressed in a previous paper /2/ where three independent approaches produced a unique value of $0.93 \mathrm{~g}$ for the mass undergoing $B \rightarrow \gamma^{\prime}$ transformation in this particular tensile specimen. Assuming that the same amount of material undergoes $\beta \rightarrow \beta^{\prime}$ transformation at higher loads, it is then possible to evaluate the molar enthalpy and entropy changes for both transformations. These results are shown in table 3 where they are compared to the values obtained from the stress-temperature equilibria (fig 3) using the clausius-Clapeyron equation.

TABLE 3-Equilibrium Temperatures, $T_{0}\left[\left(M_{s}+A_{f}\right) / 2\right]$, and Entropy and Enthalpy Changes for Alloy B obtained from Calorimetric Measurements and from $\sigma / \mathrm{T}$ Diagrams.

\begin{tabular}{|c|c|c|c|c|c|c|}
\hline & \multicolumn{3}{|c|}{$\beta \rightarrow \gamma^{1}$} & \multicolumn{3}{|c|}{$\beta \rightarrow \beta^{1}$} \\
\hline & $\mathrm{T}_{0}(\mathrm{~K})$ & $\begin{array}{c}\Delta S^{\beta+\gamma^{\prime}} \\
\left(\mathrm{Jmol}^{-1} \mathrm{~K}^{-1}\right) \\
\end{array}$ & $\begin{array}{c}\Delta \mathrm{H}^{\beta-\gamma^{\prime}}(\sigma=0) \\
\left(\mathrm{Jmol}^{-1}\right)\end{array}$ & $\mathrm{T}_{0}(\mathrm{~K})$ & $\begin{array}{c}\Delta \mathrm{S}^{\beta+\beta^{\prime}} \\
\left(\mathrm{JMOI}^{-1} \mathrm{~K}^{-1}\right) \\
\end{array}$ & $\begin{array}{c}\Delta \mathrm{H}^{\beta \rightarrow \beta^{\prime}}(\sigma=0) \\
\left(\mathrm{Jmol}^{-1}\right)\end{array}$ \\
\hline $\begin{array}{l}\text { Calorimetric } \\
\text { results }\end{array}$ & \multirow{2}{*}{$261 \pm 2$} & $\begin{array}{c}-1.54 \pm \\
0.07 \\
\end{array}$ & $-371 \pm 13$ & \multirow{2}{*}{$238 \pm 2$} & $\begin{array}{c}-1.11 \pm \\
0.10\end{array}$ & $-246 \pm 31$ \\
\hline $\begin{array}{l}\text { Values from } \\
\text { Clausius-Clapeyron } \\
\text { equation }\end{array}$ & & $\begin{array}{c}-1.01 \pm \\
0.09\end{array}$ & $-244 \pm 24$ & & $\begin{array}{c}-1.23 \pm \\
0.10\end{array}$ & $-285 \pm 30$ \\
\hline
\end{tabular}

\section{CONCLUSIONS}

1. A microcalorimeter has been designed and built which is able to operate on samples subjected to uniaxial tensile stress.

2. Stress-temperature phase diagrams have been determined for four singlecrystal Cu-Al-Ni alloys by a combination of isothermal stress-induced measurements, and thermally-induced measurements at constant stress. Such diagrams are consistent with those determined by stress-induced transformation alone, and show that the phase boundaries are co-incident for both types of measurement.

3. Thermally-induced transformation at constant stress enabled the determination of all the transformation thresholds, including those which are normally inaccessible during isothermal stress-induced experiments. 
4. The total heats exchanged during stress-free thermally-induced transformation have been measured for the four alloys. This has allowed the determination of their entropy and enthalpy changes during $B \rightarrow \gamma^{\prime}$ transformation.

5. In one of the alloys the total heats exchanged during thermally-induced transformation have been measured as a function of stress for both the $B$ $\rightarrow \gamma^{\prime}$ and $B \rightarrow B^{\prime}$ transformations. This has resulted in what appears to be the first direct measurements of the enthalpy and entropy changes during $B \rightarrow B^{\prime}$ transformation in an alloy of this general composition.

\section{ACKNOWLEDGEMENTS.}

CMF acknowledges DGICYT (Spain) for the award of a sabbatical fellowship in Barcelona. Financial support from CICYT (Spain) and from the British Council in spain is gratefully acknowledged.

\section{REFERENCES.}

/1/ Ahlers, M, Prog Mat Sci 30 (1986) 135

/2/ Ortín,J, Mañosa,Ll, Friend,CM, Planes, A, Yoshikawa, M, in print Phil Mag

/3/ Oxtín, J, Planes, A, Acta Met 37 (1989) 1433

14/ Friend, CM, Ortín,J, Planes,A, Mañosa,Ll, Yoshikawa,M, Scripta Met Mat 24 (1990) 1641

15/ Guénin,G, Macqueron, JL, Mantel,M, Auguet, C, Cesari, E, Mañosa, Ll, Planes, A, Ortín, J, Picornell,C, Seguí,C, Torra, V, Proc Int Conf on Martensitic Transformations 1986 (Nara, Japan: The Japan Inst of Metals) pp 794-799

16/ Otsuka,K, Wayman,CM, Nakai,k, Sakamoto,H, Shimizu,k, Acta Met 24 (1976) 207

/7/ otsuka,K, Sakamoto,H, Shimizu,K, Acta Met 27 (1979) 585

/8/ Salzbrenner, RJ, Cohen, M, Acta Met 27 (1979) 739

19/ Friend, CM, Scripta Met 23 (1989) 1817

/10/ Wollants, P, De Bonte, M, Roos, JR, Z Metallkde 74 (1983) 127. 